\title{
VMAT2 availability in Parkinson's disease with probable REM sleep behaviour disorder
}

Mikaeel Valli ${ }^{1,2,3^{*}}$, Sang Soo $\mathrm{Cho}^{4}$, Carme Uribe ${ }^{1}$, Mario Masellis ${ }^{3,5,6}$, Robert Chen ${ }^{2,3,7}$,

Alexander Mihaescu ${ }^{1,2,3}$ and Antonio P. Strafella ${ }^{1,2,3,7^{*}}$

\begin{abstract}
REM sleep behaviour disorder (RBD) can be an early non-motor symptom of Parkinson's disease (PD) with pathology involving mainly the pontine nuclei. Beyond the brainstem, it is unclear if RBD patients comorbid with PD have more affected striatal dopamine denervation compared to PD patients unaffected by RBD (PD-RBD-). To elucidate this, we evaluated the availability of vesicular monoamine transporter 2 (VMAT2), an index of nigrostriatal dopamine innervation, in 15 PD patients with probable RBD (PD-RBD+), 15 PD-RBD-, and 15 age-matched healthy controls (HC) using $\left[{ }^{11} \mathrm{C}\right]$ DTBZ PET imaging. This technique measured VMAT2 availability within striatal regions of interest (ROI). A mixed effect model was used to compare the radioligand binding of VMAT2 between the three groups for each striatal ROI, while co-varying for sex, cognitive function and depression scores. Multiple regressions were also computed to predict clinical measures from group condition and VMAT2 binding within all ROIs explored. We observed a significant main effect of group condition on VMAT2 availability within the caudate, putamen, ventral striatum, globus pallidus, substantia nigra, and subthalamus. Specifically, our results revealed that PD-RBD+ had lower VMAT2 availability compared to $\mathrm{HC}$ in all these regions except for the subthalamus and substantia nigra, while PD-RBD- was significantly lower than $\mathrm{HC}$ in all these regions. PD-RBD - showed a negative relationship between motor severity and VMAT2 availability within the left caudate. Our findings reflect that both PD patient subgroups had similar denervation within the nigrostriatal pathway. There were no significant interactions detected between radioligand binding and clinical scores in PD-RBD+. Taken together, VMAT2 and striatal dopamine denervation in general may not be a significant contributor to the pathophysiology of RBD in PD patients. Future studies are encouraged to explore other underlying neural chemistry mechanisms contributing to RBD in PD patients.
\end{abstract}

Keywords: Parkinson's disease, REM sleep behaviour disorder, Positron emission tomography, VMAT2, [ $\left.{ }^{11} \mathrm{C}\right] \mathrm{DTBZ}$

\section{Introduction}

Rapid eye movement (REM) sleep behaviour disorder (RBD) is a parasomnia characterized by the loss of normal skeletal muscle atonia during REM sleep [1]. This results in dream enacting behaviours that are often violent or aggressive in nature. Increasing evidence shows that the RBD pathology involves the pontine nuclei

\footnotetext{
*Correspondence: mikaeel.valli@mail.utoronto.ca; antonio.strafella@uhn.ca ${ }^{1}$ Brain Health Imaging Centre, Campbell Family Mental Health Research Institute, Centre for Addiction and Mental Health, University of Toronto, Toronto, ON, Canada

Full list of author information is available at the end of the article
}

within the brainstem [2]. Studies have further shown a link between idiopathic RBD and alpha-synucleinopathies such as Parkinson's disease (PD) [3, 4]. RBD is one of the hallmark prodromal features that manifests as early as 20 years before official PD diagnosis in many patients [5]. The risk estimate of developing PD is 33\% at 5 years since RBD diagnosis. This risk sharply increases to $91 \%$ at 14 years [6]. This underscores the importance of RBD in PD pathology as it is a strong clinical predictor of PD and is co-morbid in nearly half of the PD patient population [6].

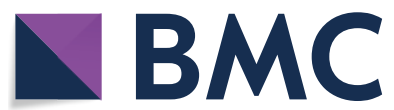

(c) The Author(s) 2021. Open Access This article is licensed under a Creative Commons Attribution 4.0 International License, which permits use, sharing, adaptation, distribution and reproduction in any medium or format, as long as you give appropriate credit to the original author(s) and the source, provide a link to the Creative Commons licence, and indicate if changes were made. The images or other third party material in this article are included in the article's Creative Commons licence, unless indicated otherwise in a credit line to the material. If material is not included in the article's Creative Commons licence and your intended use is not permitted by statutory regulation or exceeds the permitted use, you will need to obtain permission directly from the copyright holder. To view a copy of this licence, visit http://creativecommons.org/licenses/by/4.0/. The Creative Commons Public Domain Dedication waiver (http://creativeco mmons.org/publicdomain/zero/1.0/) applies to the data made available in this article, unless otherwise stated in a credit line to the data. 
At the time of PD diagnosis, there is already advanced degeneration within the nigrostriatal dopaminergic system, with estimates of $50-70 \%$ dopaminergic terminal loss within the putamen based on a post-mortem study [7] and in vivo neuroimaging of early-stage PD patients with unilateral motor impairment [8]. In the prodromal phase between the time of RBD diagnosis and clinical manifestation of $\mathrm{PD}$, there are multiple neuroimaging studies that show presynaptic striatal degeneration, particularly by indexing the dopamine transporter (DAT) density. DAT is a symporter primarily found on the presynaptic terminals of nigrostriatal neurons. It carries the function of clearing released dopamine from the synaptic cleft back into the presynaptic terminals-thereby modulating dopaminergic transmission [9]. Neuroimaging studies report consistent striatal DAT depletion in $20-40 \%$ of polysomnography confirmed idiopathic RBD patients (i.e., without PD) relative to healthy controls, particularly within the putamen $[6,10-13]$. The decline of striatal DAT has been demonstrated from healthy controls to sub-clinical RBD (REM sleep without atonia on polysomnography, but without abnormal nocturnal behaviours) to manifest RBD to PD $[14,15]$. This worsening pattern continues in a subset of PD patients with probable RBD where they show greater DAT depletion in the caudate and putamen compared to PD patients without probable RBD $[16,17]$. Consistently, another study by Arnaldi et al. (2015) showed DAT levels in the putamen progressively decreased from idiopathic RBD to PD without probable RBD to PD with probable RBD. Taken together, these studies implicated that DAT imaging may play a contributory role in detection of RBD and in PD patients with probable RBD.

In addition to measuring DAT density as an index for presynaptic dopaminergic integrity, quantification of the type 2 vesicular monoamine transporter (VMAT2) is another approach. VMAT2 is an integral membrane protein that is responsible for shuttling monoamine neurotransmitters including dopamine from the cytosol to the synaptic vesicles [9]. Evidence shows that VMAT2 is sensitive to changes in vesicular dopamine concentration [18]. However, its binding site was shown to be less sensitive to changes induced by medication or compensatory mechanisms associated with the loss of dopaminergic neurons relative to DAT [19]. Hence, quantifying VMAT2 levels allow for a more accurate measurement of the dopaminergic terminal integrity compared to measuring DAT levels [20]. VMAT2 can be measured with $\left[{ }^{11} \mathrm{C}\right]$-dihydrotetrabenazine $\left(\left[{ }^{11} \mathrm{C}\right] \mathrm{DTBZ}\right)$ or $\left[{ }^{18} \mathrm{~F}\right] \mathrm{AV}-133$ and both are reliable for in vivo imaging of VMAT2 density and distribution within the basal ganglia [9]. A recent study using $\left[{ }^{18} \mathrm{~F}\right] \mathrm{AV}-133$ showed that probable $\mathrm{RBD}$ patients had reduced VMAT2 levels within the caudate nuclei and putamen relative to healthy controls [21]. Similar results were shown in a smaller older study using $\left[{ }^{11} \mathrm{C}\right] \mathrm{DTBZ}$ where the authors observed reduced VMAT2 availability in the posterior putamen in polysomnography confirmed RBD patients in relation to healthy controls [22]. As presented here, there are limited studies that focused on VMAT2 imaging, but these investigations indicate that VMAT2 imaging may also have a certain role in detecting RBDs.

In summary, while the literature seems to suggest an association of RBD with the presynaptic dopaminergic system, it remains unclear if PD patients with RBD have more extensive striatal dopamine denervation than $\mathrm{PD}$ patients without RBD. Furthermore, the characterization of VMAT2 has been rarely explored-especially in PD patients with RBD. By examining VMAT2 as an in vivo molecular target with its unique properties, we aim to elucidate VMAT2 levels in PD patients who are comorbid with probable RBD. We achieved this by using $\left[{ }^{11} \mathrm{C}\right]$ DTBZ PET imaging in PD patients with probable RBD and in PD patients without probable RBD, while having age-matched healthy controls. We hypothesized that PD patients with probable RBD to show more extensive dopamine denervation pathology, that is reduced VMAT2 availability, in striatal regions including the caudate, putamen, and ventral striatum relative to $P D$ patients without probable RBD and healthy controls. This hypothesis would be consistent with reductions of the DAT observed in the striatal regions reported in PD patients with probable RBD.

\section{Methods \\ Participants}

A total of 45 participants were enrolled in this study: 15 PD patients without probable RBD, 15 PD patients with probable RBD, and 15 age-matched healthy controls (Table 1). Some of the imaging, demographic, cognitive and psychological data from these participants have been reported previously [23, 24]. Both PD patient groups were diagnosed based on the UK Parkinson Disease Society Brain Bank criteria. All participants had no evidence of other neurological or psychiatric conditions or any other medical conditions that precluded them from the PET and MR imaging. The severity of PD motor symptoms was tested through the Hoehn and Yahr Scale and the United Parkinson Disease Rating Scale (UPDRS-III) while patients were on medication. Levodopa equivalent daily dose (LEDD) calculation for each patient has been previously described by Evans et al. [25]. All participants were age-matched; and PD patients were matched for disease severity based on UPDRS-III score and LEDD.

PD patients were screened for RBD as part of their routine neurology clinic visits. This screening was done 
Table 1 Demographic, behavioural, clinical and PET imaging characteristics of participants

\begin{tabular}{|c|c|c|c|c|}
\hline & $\mathrm{HC}$ & PD-RBD- & PD-RBD+ & $p$ value \\
\hline$N(\mathrm{M}: \mathrm{F})$ & $15(3: 12)$ & $15(8: 7)$ & $15(10: 5)$ & $0.03^{\mathrm{a}}$ \\
\hline Age [years] $\pm S D$ (range) & $67.1 \pm 5.14(58-79)$ & $70.7 \pm 5.67(60-80)$ & $68.1 \pm 6.48(56-80)$ & 0.23 \\
\hline $\mathrm{BDI} \pm \mathrm{SD}$ & $2.33 \pm 1.29$ & $3.00 \pm 1.36$ & $5.00 \pm 4.32$ & 0.03 \\
\hline $\mathrm{MoCA} \pm \mathrm{SD}$ & $27.6 \pm 2.13$ & $24.93 \pm 2.93$ & $23.87 \pm 3.24$ & 0.002 \\
\hline Disease duration [years] $\pm S D$ & - & $7.20 \pm 4.49$ & $6.76 \pm 3.67$ & 0.77 \\
\hline UPDRS-III $\pm S D$ & - & $28.53 \pm 17.18$ & $23.87 \pm 10.84$ & 0.38 \\
\hline Hoehn and Yahr Score \pm SD & - & $2.20 \pm 0.41$ & $2.13 \pm 0.39$ & 0.68 \\
\hline $\operatorname{LEDD}[\mathrm{mg}] \pm S D$ & - & $701.70 \pm 522.04$ & $723.45 \pm 410.75$ & 0.90 \\
\hline$\left[{ }^{11} \mathrm{C}\right] \mathrm{DTBZ}$ dose $[\mathrm{mCi}] \pm S \mathrm{D}$ & $9.54 \pm 0.87$ & $9.33 \pm 0.59$ & $9.72 \pm 0.45$ & 0.29 \\
\hline$\left[{ }^{11} \mathrm{C}\right] \mathrm{DTBZ}$ mass $[\mu \mathrm{g}] \pm \mathrm{SD}$ & $1.84 \pm 1.78$ & $1.28 \pm 0.53$ & $1.74 \pm 1.31$ & 0.48 \\
\hline $\begin{array}{l}{\left[{ }^{11} \mathrm{C}\right] \mathrm{DTBZ} \text { specific activity [mCi/ }} \\
\mu \mathrm{mol}] \pm S D\end{array}$ & $2529.77 \pm 1312.82$ & $2658.75 \pm 995.19$ & $2319.78 \pm 816.03$ & 0.68 \\
\hline
\end{tabular}

BDI, Beck Depression Inventory; HC, healthy controls; LEDD, levodopa equivalent daily dose (calculated according to Evans et al. [25]); MoCA, Montreal Cognitive Assessment; PD-RBD+, PD patients with probable RBD; PD-RBD-, PD patients without probable RBD; UPDRS-III, Unified Parkinson's Disease Rating Scale III

a Pearson Chi-Square

prior to any imaging data collection, including patients reported previously by our group [23, 24]. Identification of probable RBD symptoms was completed through using the informant-based response to the first question on the Mayo Sleep Questionnaire: "Have you ever seen the patient appear to act out his/her dreams while sleeping." Patients were classified as clinically probable RBD if the patients' sleeping partner answered yes to this question [26]. This singular question has been validated against polysomnography, with a sensitivity of $98 \%$ and specificity of $74 \%$, in a multicenter prospective cohort study of healthy older adults and suspected neurodegenerative disease [26, 27].

In order to prepare for the PET scan, PD patients performed an overnight 12-h withdrawal from anti-parkinsonian medication to minimize the effect of medication during the scans while maintaining patient comfort and functioning [28]. To prevent excessive fatigue, the PET and structural MRI scans were completed on separate days. Montreal Cognitive Assessment (MoCA; [29]) and Beck Depression Inventory (BDI; [30] were obtained on all participants to assess general cognitive capabilities and depression levels, respectively. All participants provided informed written consent prior to beginning any imaging study procedures which were approved by the research ethics committees for the Centre of Addictions and Mental Health and the University Health Network of the University of Toronto.

\section{Imaging acquisition}

The preparation of the $\left[{ }^{11} \mathrm{C}\right] \mathrm{DTBZ}$ radioligand was described previously [31]. PET scans were collected using a three-dimensional (3D) high resolution research tomograph (HRRT) scanner (Siemens, Knoxville, TN). This equipment allows the measurement of radioactivity in 207 brain slices, with a thickness of $1.22 \mathrm{~mm}$ each [23]. The detectors of the HRRT are a lutetium oxyorthosilicate/lutetium-yttrium oxyorthosilicate phoswich, with each crystal element measuring $2 \times 2 \times 10$ $\mathrm{mm}^{3}$. To minimize head motion, a customized thermoplastic facemask was provided to each participant prior to the HRRT PET scan, and the facemask was secured through the head-fixation system (Tru-Scan Imaging, Annapolis). After securing participants within the PET scanner, a transmission scan was first completed using a single photon point source, ${ }^{137} \mathrm{Cs}\left(\mathrm{t}_{1 / 2}=30.2\right.$ years, $\mathrm{E}_{\gamma}=662 \mathrm{keV}$ ), which had a duration of 6 min and $9 \mathrm{~s}$. This transmission scan was immediately followed by the acquisition of the emission scan to correct for attenuation (where frame durations were: $1 \times$ background; 15 frames $\times 60 \mathrm{~s}$; and 15 frames $\times 300 \mathrm{~s}$ ). Subsequently, the $\left[{ }^{11} \mathrm{C}\right]$ DTBZ radioligand was injected as a bolus into an intravenous line placed in the antecubital vein. Emission data were collected in list mode for 60 min while subjects were at rest.

The emission list mode data were re-binned into a series of 3D sinograms. The 3D sinograms were gap filled, scatter corrected and Fourier re-binned into 2-dimensional (2D) sinograms. The images were reconstructed from the $2 \mathrm{D}$ sinograms using a $2 \mathrm{D}$ filtered-back projection algorithm. The reconstructed images had $256 \times 256 \times 207$ cubic voxels that measured $1.22 \times 1.22 \times 1.22 \mathrm{~mm}^{3}$. The dynamic images were then reconstructed into 17 frames. The first frame was variable as it was dependent on the time between the start of acquisition and the introduction of the $\left[{ }^{11} \mathrm{C}\right]$ 
DTBZ radioligand in the tomograph field of view. The following frames were defined as: $1 \times \geq 22 \mathrm{~s}, 4 \times 60 \mathrm{~s}$, $3 \times 120 \mathrm{~s}, 8 \times 300 \mathrm{~s}$, and $1 \times 600 \mathrm{~s}$.

To provide anatomical reference for the parametric PET image analysis and to rule out structural lesions, a whole-brain T1-weighted MR image was acquired from each participant using GE Signa $\mathrm{HD} \times$ MRI system (GE Discovery MR750 $3 \mathrm{~T}$; T1-weighted images, fast spoiled gradient echo with repletion time $=6.7 \mathrm{~ms}$, echo time $=3.0 \mathrm{~ms}$, flip angle $=8 \mathrm{~mm}$, slice thickness $=1 \mathrm{~mm}$, number of excitations $=1$, and matrix size $=256 \times 192$ ).

\section{Imaging analysis}

Image preprocessing was completed using an in-house software, Regions of Mental Interest (ROMI; [32]). This software was used to obtain the time activity curve (TAC) for the reference region-the occipital lobe-for all participants. ROMI used Statistical Parametric Mapping (SPM8, Welcome Department of Imaging Neuroscience, London, UK), where each participant's MR image was used to nonlinearly transform a standardized brain template (International Consortium for Brain Mapping/ Montreal Neurological Institute $152 \mathrm{MRI}$ ) with predefined regions of interests (ROIs). The individual ROI template underwent further refinement based on the gray matter probability of the segmented MRI. The refinement of each individual's ROIs were then aligned and resliced using a normalized mutual information algorithm [33] to match the individuals PET scan. Subsequently, the TAC for the occipital lobe was obtained from the dynamic $\left[{ }^{11} \mathrm{C}\right] \mathrm{DTBZ}$ PET image in the native space.

Upon the completion of the pre-registration procedure with ROMI, $\left[{ }^{11} \mathrm{C}\right] \mathrm{DTBZ}$ PET parametric non-displaceable binding potential $\left(\mathrm{BP}_{\mathrm{ND}}\right)$ maps were generated in the native PET space with simplified reference tissue model [34] using the occipital cortex TAC value as reference region (obtained through ROMI). This was completed using Receptor Parametric Mapping software (RPM; [35]) within MATLAB R2015a (version 8.5.0.197613; MathWorks). Using SPM12 (version 7487) within MAT$\mathrm{LAB}$, the parametric $\mathrm{BP}_{\mathrm{ND}}$ images were transformed into standardized stereotaxic space using each participants' individual MRI. These normalized images were then smoothed with a Gaussian function at $8 \mathrm{~mm}$ full width half-maximum.

The ROIs we examined were caudate, putamen, internal globus pallidus, external globus pallidus, substantia nigra, and subthalamus. These ROIs were obtained from the WFU-PickAtlas toolbox (http://www.fmri.wfubmc. edu/cms/software). In addition, we examined associative striatum, motor striatum, and ventral striatum-which were delineated according to previously specified criteria [36]. These ROIs were transformed into a parametric $\left[{ }^{11} \mathrm{C}\right] \mathrm{DTBZ}$ PET $\mathrm{BP}_{\mathrm{ND}}$ map, and the $\mathrm{BP}_{\mathrm{ND}}$ values were extracted using MATLAB based REX toolbox (http:// web.mit.edu/swg/software.htm). We used the $\mathrm{BP}_{\mathrm{ND}}$ values obtained through REX for each ROI for statistical analysis.

\section{Statistical analysis}

Demographic characteristics were tested for differences between the three groups (i.e., healthy controls, $\mathrm{PD}$ patients without probable RBD, and $\mathrm{PD}$ patients with probable RBD) using ANOVA. Specifically, we performed ANOVA and Bonferroni post-hoc testing on age, MoCA score, BDI, UPDRS-III score, Hoehn and Yahr score, LEDD amount, quality and quantity of injected radioligand across all three participant groups. To assess for differences in sex proportions between groups, a chisquared analysis was performed. Statistical outliers was investigated using the interquartile range method [37].

Mixed effects model was used to compare the extracted $\left[{ }^{11} \mathrm{C}\right] \mathrm{DTBZ} \mathrm{BP}_{\mathrm{ND}}$ between the three groups for each ROI. The fixed factors within the model were group (i.e., healthy controls, PD patients without probable RBD, and PD patients with probable RBD) and side (i.e., left vs. right ROI); the participants were kept as the random factor. The model also co-varied for sex, MoCA and BDI score. Post hoc independent sample $t$ tests were used to assess for differences between groups and were corrected for multiple comparisons using the Bonferroni method.

Multiple regression analyses were used to correlate $\left[{ }^{11} \mathrm{C}\right] \mathrm{DTBZ} \mathrm{BP}_{\mathrm{ND}}$ within each ROI against clinical measures including UPDRS-III score, Hoehn and Yahr score, LEDD amount, and disease duration while factoring patient group condition (i.e., PD patients with and without probable RBD). This regression model included sex, MoCA and BDI as co-variates. All tests were completed using SPSS (version 21; Chicago, IL); and the alpha level was set to 0.05 as a cut-off to determine significance.

\section{Results}

The demographic, clinical and psychological characteristics for each group are summarized in Table 1. There were no differences between all participant groups (i.e., healthy controls, PD patients without probable RBD, and PD patients with probable RBD) for age, radiotracer injected dose, injected mass, and specific activity. PD patients with and without probable RBD were comparable in relation to their disease duration, UPDRS-III score, Hoehn and Yahr score, and LEDD amount. However, differences were observed between groups for sex, MoCA, and BDI. These three variables were included as co-variates in subsequent analyses. There were no statistical outliers using the 1.5 interquartile range method. 
As there were main effects of $\operatorname{MoCA}\left[\mathrm{F}_{(2,42)}=7.29\right.$, $p=0.002]$ and $\mathrm{BDI}\left[\mathrm{F}_{(2,42)}=3.89, p=0.028\right]$, we followed up with Bonferroni post hoc $t$-tests to detect where the group differences occurred. These particular results were reported previously by Valli et al. [24]. In summary for $\mathrm{BDI}$, patients with probable RBD had the highest depression score, then PD patients without probable RBD, then healthy controls. Only PD patients with probable RBD were statistically higher than healthy controls $(t=-2.68$, $p=0.03$ ). Regarding MoCA, in comparison to healthy controls, PD patients with probable RBD had the lowest cognitive score $(t=3.70, p=0.003)$, followed by patients without probable $\operatorname{RBD}(t=2.66, p=0.0165)$. PD patients with probable RBD had relatively lower MoCA score compared to PD patients without probable RBD but this difference did not reach significance.

Results from the mixed effect model, co-varying for sex, MoCA and BDI score, revealed significant main effect of group on $\left[{ }^{11} \mathrm{C}\right] \mathrm{DTBZ}$ binding within all ROIs explored: caudate $\left[\mathrm{F}_{(2,39)}=10.6, p<0.001\right]$, putamen $\left[\mathrm{F}_{(2 \text {, }}\right.$ 39) $=41.57, p<0.001]$, associative striatum $\left[\mathrm{F}_{(2,39)}=17.49\right.$, $p<0.001]$, motor striatum $\left[\mathrm{F}_{(2,39)}=53.49, p<0.001\right]$, ventral striatum $\left[\mathrm{F}_{(2,39)}=7.42, p=0.002\right]$, external globus pallidus $\left[\mathrm{F}_{(2,39)}=29.17, p<0.001\right]$, internal globus pallidus $\left[\mathrm{F}_{(2,39)}=7.33, p=0.002\right]$, substantia nigra $\left[\mathrm{F}_{(2,}\right.$, $\left.{ }_{39)}=7.58, p=0.002\right]$, and subthalamus $\left[\mathrm{F}_{(2,39)}=4.61\right.$, $p=0.016]$. Specifically, we found that the mean $\left[{ }^{11} \mathrm{C}\right]$ DTBZ $\mathrm{BP}_{\mathrm{ND}}$ of PD patients without probable RBD were lower than healthy controls for all these significant ROIs. Similarly, PD patients with probable RBD had reduced $\mathrm{BP}_{\mathrm{ND}}$ compared to healthy controls for all these significant ROIs except for the subthalamus and substantia nigra. Summary of the Bonferroni corrected post-hoc $t$-test results between PD patients without probable RBD versus healthy controls and $\mathrm{PD}$ patients with probable RBD versus healthy controls is displayed in Table 2 . We were not able to detect statistical differences between PD patients with and without probable RBD for these significant regions (Fig. 1). In summary, the significant findings from the mixed effects model was driven mainly by the differences between healthy controls and both PD subgroups, not the differences between PD patients with and without probable RBD.

Multiple regressions were computed to predict clinical measures (i.e., disease duration, UPDRS-III score, Hoehn and Yahr score, and LEDD amount) from group condition (i.e., PD patients with and without probable RBD), and $\left[{ }^{11} \mathrm{C}\right] \mathrm{DTBZ} \mathrm{BP}_{\mathrm{ND}}$ of all basal ganglia ROIs explored, while covarying for sex, MoCA and BDI score. We were able to observe a significant interaction between left caudate $\mathrm{BP}_{\mathrm{ND}}$ and group condition where these two measures predicted UPDRS-III score $\left(\mathrm{F}_{(1,23)}=11.13, p=0.003\right.$, $\mathrm{R}^{2}=0.59$ ). Figure 2 displays the interaction plot between
Table 2 Post-hoc $t$-test results looking at $\left[{ }^{11} \mathrm{C}\right] \mathrm{DTBZ} \quad \mathrm{BP}_{\mathrm{ND}}$ differences between PD-RBD- vs. healthy controls and PD-RBD+vs. healthy controls

\begin{tabular}{|c|c|c|c|c|}
\hline \multirow[t]{2}{*}{ Basal ganglia ROIs } & \multicolumn{2}{|c|}{$\begin{array}{l}\text { (A) PD-RBD-vs } \\
\text { healthy controls }\end{array}$} & \multicolumn{2}{|c|}{$\begin{array}{l}\text { (B) PD-RBD+vs } \\
\text { healthy controls }\end{array}$} \\
\hline & $t$ value & $p$ value & $t$ value & $p$ value \\
\hline Caudate & 4.38 & $<0.001$ & 3.72 & 0.002 \\
\hline Putamen & 8.44 & $<0.001$ & 7.76 & 0.001 \\
\hline Subthalamus & 3.02 & 0.013 & 1.96 & 0.168 \\
\hline Ventral striatum & 3.77 & 0.002 & 2.84 & 0.021 \\
\hline Associative striatum & 5.59 & $<0.001$ & 4.84 & $<0.001$ \\
\hline Motor striatum & 9.51 & $<0.001$ & 8.89 & $<0.001$ \\
\hline External globus pallidus & 6.92 & $<0.001$ & 6.68 & $<0.001$ \\
\hline Internal globus pallidus & 3.33 & 0.006 & 3.49 & 0.04 \\
\hline Substantia nigra & 3.90 & 0.001 & 2.35 & 0.072 \\
\hline
\end{tabular}

This table displays the Bonferroni corrected post-hoc $t$-test results for significant ROIs detected through the mixed effects model. Column A on the left displays results looking at $\left[{ }^{11} \mathrm{C}\right] \mathrm{DTBZ} \mathrm{BP}_{\mathrm{ND}}$ differences between $\mathrm{PD}$ patients without probable RBD (PD-RBD-) and healthy controls. Column $B$ on the right shows results between PD patients with probable RBD (PD-RBD+) and healthy controls

left caudate $\mathrm{BP}_{\mathrm{ND}}$ and UPDRS-III score where there is a steep negative correlation for PD patients without probable RBD. This relationship was not present for PD patients with probable RBD. No other significant interactions were detected through the multiple regression analyses between the $\mathrm{BP}_{\mathrm{ND}}$ of the significant ROIs and group condition on clinical scores.

\section{Discussion}

This study aimed to characterize whether PD patients with probable RBD had more extensive striatal dopamine denervation pathology relative to $\mathrm{PD}$ patients without RBD. We observed that both PD patient subgroups had lower tracer binding compared to healthy controls within the basal ganglia. There were no indications to show $\left[{ }^{11} \mathrm{C}\right] \mathrm{DTBZ}$ binding differences between PD patients with and without probable RBD, which did not confirm our hypothesis. The reduced radioligand signal compared to healthy controls reflects lower VMAT2 availability in both patient subgroups-which implies nigrostriatal denervation as a result of PD neurodegeneration. In the group of PD patients without probable RBD, there was a strong negative relationship between left caudate VMAT2 availability and UPDRS-III score. In other words, with the worsening of motor severity in patients without probable RBD, it correlated with more presynaptic denervation in the left caudate. However, this relationship was not present in PD patients with probable RBD.

The observed reduction of VMAT2 density as measured by $\left[{ }^{11} \mathrm{C}\right] \mathrm{DTBZ}$ in our PD patient sample in the 

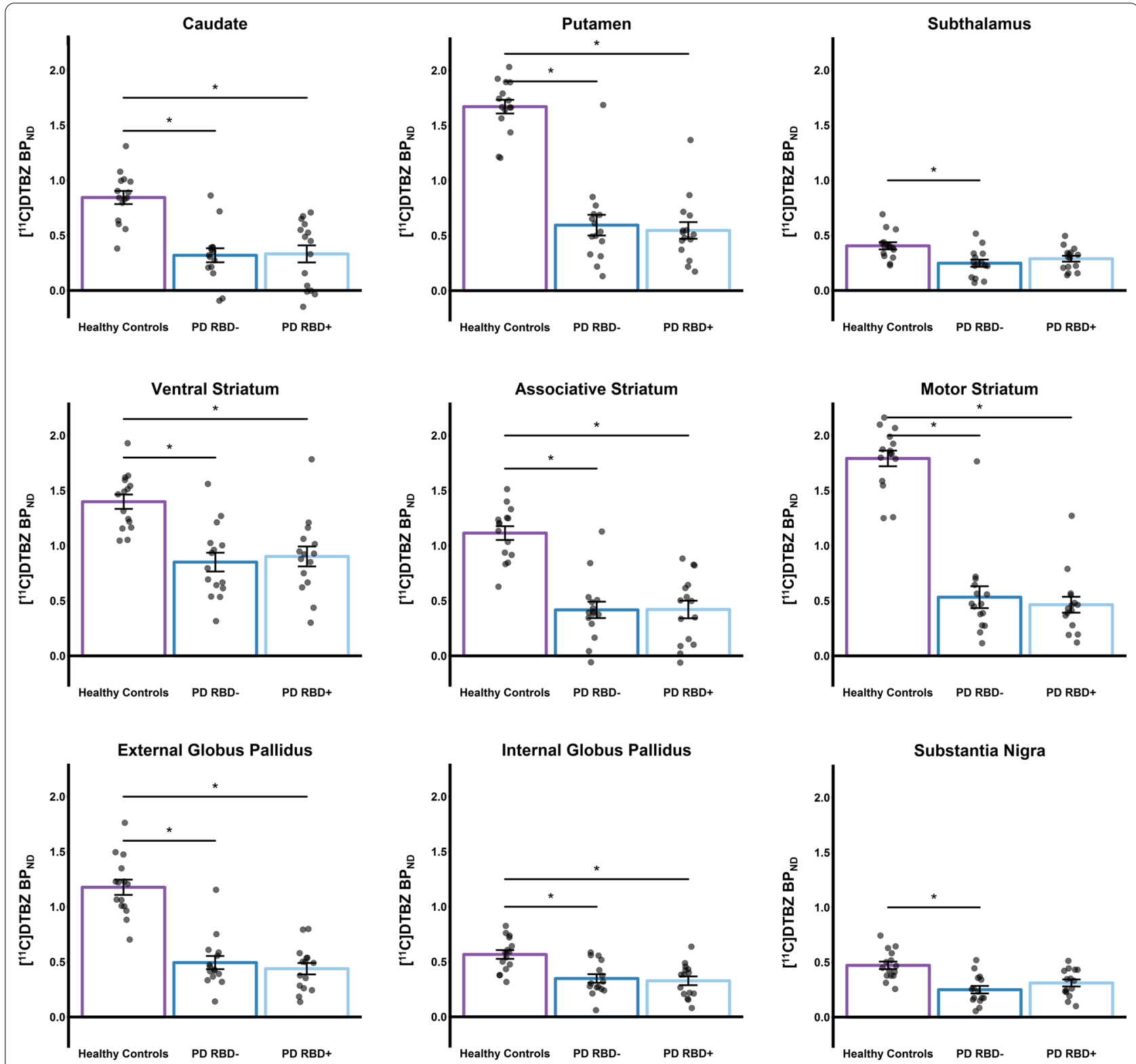

Fig. 1 This figure displays the $B_{N D}$ differences between groups of healthy controls, PD patients without probable RBD (PD-RBD-), and PD patients with probable RBD (PD-RBD+) in all explored regions within the basal ganglia. All regions revealed to have significant main effect. $\left[{ }^{11} \mathrm{C}\right] \mathrm{DTBZ} \mathrm{BP} \mathrm{ND}_{\mathrm{N}}$ represents the degree of VMAT2 availability. We found that $\mathrm{BP}_{\mathrm{ND}}$ of PD-RBD - was reduced compared to healthy controls in all regions shown. This pattern was similarly seen for PD-RBD+ in relation to healthy controls for all regions except for the subthalamus and substantia nigra. ${ }^{*} p<0.05$, Bonferroni corrected

putamen, caudate, substantia nigra, and globus pallidus relative to controls is consistent with previous neuroimaging studies of VMAT2 [8, 38-41]. This reinforces the notion that $\left[{ }^{11} \mathrm{C}\right] \mathrm{DTBZ}$ PET imaging holds the potential to effectively differentiate PD patients from controls [42, 43]. The observed significant correlation between motor severity for PD patients and left caudate
$\mathrm{BP}_{\mathrm{ND}}$ was also consistent with previous studies in their sample of PD patients with no reports of other comorbidities $[42,43]$. This relationship is in line with the view that motor disability in PD is primarily associated with impairment of subcortical structures within the nigrostriatal pathway [44]. In contrast, no significant interactions were observed between radiotracer binding and clinical measures in PD patients with probable 


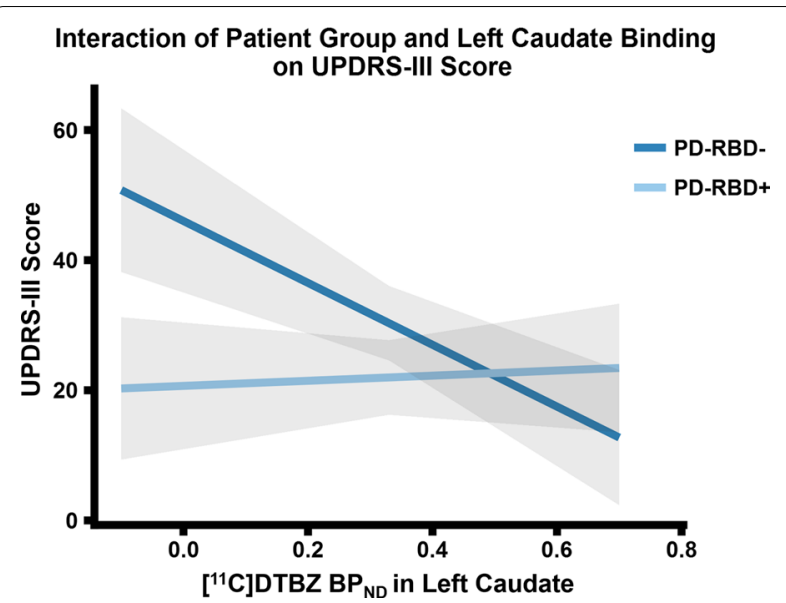

Fig. 2 This interaction plot is a result from the regression analysis. Patient group moderates the left caudate $\mathrm{BP}_{\mathrm{ND}}$ in $\mathrm{PD}$ patients without probable RBD (PD-RBD-): as the UPDRS-III score increases, PD-RBD - patients have lower VMAT2 availability. However, this relationship is non-existent for PD patients with probable RBD (PD-RBD+). This figure was plotted using marginal means that accounted for the included co-variates: sex, MoCA and BDI. The grey band for each line represents the $95 \%$ confidence interval

RBD, thus implying that other neurochemical abnormalities may account for this clinical complication.

Unlike previous DAT studies [15, 17, 45], we found no evidence of VMAT2 level differences between groups of PD patients with and without probable RBD. This observation is consistent with a previous investigation that used $\left[{ }^{11} \mathrm{C}\right] \mathrm{DTBZ}$ PET imaging as a secondary focus to their study objectives in PD patients with and without probable RBD [46]. Specifically, they were unable to differentiate VMAT2 levels of PD patients with probable RBD from PD patients without probable RBD within the caudate and putamen [46]. Our current study was different from this previous study where we used age-matched healthy controls and explored more brain regions within the basal ganglia, beyond just the caudate and putamen. There are several possible reasons that could explain the lack of differences in VMAT2 levels between PD patients with and without probable RBD that we observed. VMAT2 has been demonstrated to be a stable marker for presynaptic nigrostriatal integrity as it is less prone to changes induced by medications or compensatory mechanisms associated with the loss of dopaminergic neurons [19]. Our findings reflect that VMAT2 remained unaffected by the co-morbidity of probable RBD in conjunction with presynaptic changes associated with medication or compensatory mechanisms that would normally occur in PD [19]. This implies that the PD pathophysiology is the primary driving force depleting VMAT2 availability in both groups.
An alternative explanation may involve the relationship between the activities and availability of VMAT2 and DAT in the presynaptic terminals [47]. Previous literature has consistently shown that PD patients with probable RBD have lower DAT availability relative to $\mathrm{PD}$ patients and controls $[15,17,45]$. This observed reduction of DAT levels would in turn lower the neural ability to reuptake dopamine back into the presynaptic terminals for vesicular repackaging and subsequent reutilization $[18,48,49]$. The lower levels of presynaptic vesicles with dopamine should in turn result in a reduction of the VMAT2 levels in PD with probable RBD. However, this rationale was defied by the lack of differences in VMAT2 levels between PD patients with and without probable RBD. It could be possible that there were varying endogenous intravesicular dopamine levels in PD patients with and without probable RBD that may have netted in negligible differences in $\mathrm{BP}_{\mathrm{ND}}$ signalling between the two groups [18].

Previous literature shows other molecular structures and neurotransmitters that may play a larger role contributing to RBD in PD apart from DAT [15, 17, 45]. Outside the striatum, our group showed a negative relationship where D2 receptor availability within the uncus parahippocampus decreased with increasing disease severity in PD patients with probable RBD relative to PD patients without probable RBD [24]. Other studies showed altered cholinergic [46] and noradrenaline levels [50], along with changes in glucose metabolic activity [16] in PD patients with probable RBD relative to patients without probable $\mathrm{RBD}$, suggesting RBD pathophysiology in PD is multisystemic that impacts regions beyond the striatum and the dopaminergic system.

This study has some limitations to consider. Our patients were screened for probable RBD through the first question of the Mayo Sleep Questionnaire as part of their routine neurology clinic visit. These patients were not confirmed through the sleep polysomnography test. Despite this, the questionnaire has been validated in two studies. The first study validated the questionnaire against polysomnography in a multi-centre prospective cohort trial that included patients suspected to have neurodegenerative disease and healthy older adults. This study achieved a sensitivity of $98 \%$ and specificity of $74 \%$ [26]. A follow up validation study of the Mayo Sleep Questionnaire was carried out in a community-based sample of 128 participants who had underwent a previous polysomnography test, and resulted in a sensitivity of $100 \%$ and specificity of $95 \%$ [27]. These evidences makes the sleep questionnaire a useful research tool in settings where polysomnography is not readily available [51].

Another limitation is that patients were assessed on several clinical measures while "ON" dopamine 
replacement therapy and they were "OFF" medication during the PET scan. In turn, the relationship between motor severity and dopaminergic degeneration within the basal ganglia may not be fully representative even though VMAT2 was shown to be less prone to medication influences [38]. Although we showed a relationship between nigrostriatal innervation and motor score in PD patients without probable $\mathrm{RBD}$, investigating motor features while "ON" and "OFF" medication and changes with VMAT2 levels may provide more insight about the true relationship observed between PD with and without probable RBD and motor severity in relation to the left caudate VMAT2 availability.

\section{Conclusion}

In comparison to age-matched controls, the current study revealed that PD patients with and without probable RBD had lower VMAT2 levels within the subcortical structures of the basal ganglia which reflects denervation within the nigrostriatal pathway. No significant interactions were detected between the $\mathrm{BP}_{\mathrm{ND}}$ and clinical scores in PD patients with probable RBD. These findings collectively suggest that VMAT2 and striatal dopamine denervation in general may not be a significant contributor to the pathophysiology of RBD in PD patients. Future studies are encouraged to explore other underlying neural chemistry mechanisms to better understand the driving force of RBD in PD patients.

\section{Acknowledgements}

The authors thank Alvina Ng, Laura Nguyen and Anusha Ravichandran for their technical assistance. The authors also thank Marcos Sanches for his invaluable assistance with the statistical analyses.

\section{Authors' contributions}

Conceptualization: MV and APS; Methodology: MV and APS; Software: MV; Formal Analysis: MV and SSC; Investigation: MV; Writing —Original Draft: MV; Writing - Review \& Editing: MV, SSC, CU, MM, RC, AM and APS; Visualization: MV; Supervision: APS; Funding Acquisition: APS. All authors read and approved the final manuscript.

\section{Funding}

This work was supported by Canadian Institutes of Health Research (CIHR) (MOP 136778). APS was supported by the Canada Research Chair program. MV was supported by the CIHR's Doctoral Award program.

\section{Availability of data and materials}

The datasets used and/or analysed during the current study are available from the corresponding author on reasonable request.

\section{Declarations}

Ethics approval and consent to participate

All participants provided informed written consent prior to beginning study procedures which were approved by the research ethics committees for the Centre of Addictions and Mental Health and the University Health Network of the University of Toronto.

\section{Consent for publication}

Not applicable.

\section{Competing interests}

Antonio Strafella is a consultant for Hoffman La Roche; received honoraria from GE Health Care Canada LTD, Hoffman La Roche.

\section{Author details}

${ }^{1}$ Brain Health Imaging Centre, Campbell Family Mental Health Research Institute, Centre for Addiction and Mental Health, University of Toronto, Toronto, ON, Canada. ${ }^{2}$ Division of Brain, Imaging and Behaviour - Systems Neuroscience, Krembil Brain Institute, UHN, University of Toronto, Toronto, ON, Canada. ${ }^{3}$ Institute of Medical Science, University of Toronto, Toronto, ON Canada. ${ }^{4}$ Department of Brain and Cognitive Science, Seoul National University, Seoul, Republic of Korea. ${ }^{5}$ Hurvitz Brain Sciences Program, Sunnybrook Research Institute, Toronto, ON, Canada. ${ }^{6}$ Division of Neurology, Department of Medicine, University of Toronto, Toronto, ON, Canada. ${ }^{7}$ Edmond J. Safra Parkinson Disease Program \& Morton and Gloria Shulman Movement Disorder Unit, Neurology Division, Dept. of Medicine, Toronto Western Hospital, UHN, University of Toronto, Toronto, ON, Canada.

Received: 16 September 2021 Accepted: 29 October 2021

Published online: 10 November 2021

\section{References}

1. Schenck CH, Bundlie SR, Ettinger MG, Mahowald MW. Chronic behavioral disorders of human REM sleep: a new category of parasomnia. Sleep. 1986;9(2):293-308.

2. Boeve BF, Silber MH, Saper CB, Ferman TJ, Dickson DW, Parisi JE, et al. Pathophysiology of REM sleep behaviour disorder and relevance to neurodegenerative disease. Brain. 2007;130(11):2770-88.

3. Iranzo A, Molinuevo JL, Santamaría J, Serradell M, Martí MJ, Valldeoriola F, et al. Rapid-eye-movement sleep behaviour disorder as an early marker for a neurodegenerative disorder: a descriptive study. Lancet Neurol. 2006;5(7):572-7.

4. Boeve BF. REM sleep behavior disorder: Updated review of the core features, the REM sleep behavior disorder-neurodegenerative disease association, evolving concepts, controversies, and future directions. Ann NY Acad Sci. 2010;1184:15-54.

5. Fereshtehnejad SM, Yao C, Pelletier A, Montplaisir JY, Gagnon JF, Postuma RB. Evolution of prodromal Parkinson's disease and dementia with Lewy bodies: a prospective study. Brain. 2019;142(7):2051-67.

6. Yousaf T, Pagano G, Wilson H, Politis M. Neuroimaging of sleep disturbances in movement disorders. Front Neurol. 2018;9(767):1-19.

7. Scherman D, Desnos C, Darchen F, Pollak P, Javoy-Agid F, Agid Y. Striatal dopamine deficiency in Parkinson's disease: role of aging. Ann Neurol. 1989;26(4):551-7.

8. Lee CS, Samii A, Sossi V, Ruth TJ, Schulzer M, Holden JE, et al. In vivo positron emission tomographic evidence for compensatory changes in presynaptic dopaminergic nerve terminals in Parkinson's disease. Ann Neurol. 2000;47(4):493-503.

9. de Natale ER, Niccolini F, Wilson H, Politis M. Molecular imaging of the dopaminergic system in idiopathic Parkinson's disease. In: Politis M, editor. International review of neurobiology. Boca Raton: Academic Press Inc.; 2018. p. 131-72.

10. Rupprecht S, Walther B, Gudziol H, Steenbeck J, Freesmeyer M, Witte $\mathrm{OW}$, et al. Clinical markers of early nigrostriatal neurodegeneration in idiopathic rapid eye movement sleep behavior disorder. Sleep Med. 2013;14(11):1064-70.

11. Eisensehr I, Linke R, Noachtar S, Schwarz J, Gildehaus FJ, Tatsch K. Reduced striatal dopamine transporters in idiopathic rapid eye movement sleep behaviour disorder. Comparison with Parkinson's disease and controls. Brain. 2000;123(6):1155-60

12. Stiasny-Kolster K, Doerr Y, Möller JC, Höffken H, Behr TM, Oertel WH, et al. Combination of "idiopathic" REM sleep behaviour disorder and olfactory dysfunction as possible indicator for a-synucleinopathy demonstrated by dopamine transporter FP-CIT-SPECT. Brain. 2005;128(1):126-37.

13. Iranzo A, Lomeña F, Stockner H, Valldeoriola F, Vilaseca I, Salamero M, et al. Decreased striatal dopamine transporter uptake and substantia nigra hyperechogenicity as risk markers of synucleinopathy in patients with idiopathic rapid-eye-movement sleep behaviour disorder: a prospective study. Lancet Neurol. 2010;9(11):1070-7. 
14. Eisensehr I, Linke R, Tatsch K, Kharraz B, Gildehaus JF, Wetter CT, et al. Increased muscle activity during rapid eye movement sleep correlates with decrease of striatal presynaptic dopamine transporters. IPT and IBZM SPECT imaging in subclinical and clinically manifest idiopathic REM sleep behavior disorder, Parkinson's disease, and controls. Sleep. 2003;26(5):507-12.

15. Arnaldi D, De Carli F, Picco A, Ferrara M, Accardo J, Bossert I, et al. Nigrocaudate dopaminergic deafferentation: a marker of REM sleep behavior disorder? Neurobiol Aging. 2015;36(12):3300-5.

16. Arnaldi D, Morbelli S, Brugnolo A, Girtler N, Picco A, Ferrara M, et al. Functional neuroimaging and clinical features of drug naive patients with de novo Parkinson's disease and probable RBD. Park Relat Disord. 2016;29:47-53. https://doi.org/10.1016/j.parkreldis.2016.05.031.

17. Chung SJ, Lee Y, Lee JJ, Lee PH, Sohn YH. Rapid eye movement sleep behaviour disorder and striatal dopamine depletion in patients with Parkinson's disease. Eur J Neurol. 2017;24(10):1314-9.

18. De La Fuente-Fernández R, Furtado S, Guttman M, Furukawa Y, Lee CS, Calne DB, et al. VMAT2 binding is elevated in dopa-responsive dystonia: visualizing empty vesicles by PET. Synapse. 2003;49(1):20-8.

19. Arena JE, Stoessl AJ. Optimizing diagnosis in Parkinson's disease: radionuclide imaging. Park Relat Disord. 2016;22:S47-51.

20. Frey KA, Koeppe RA, Kilbourn MR, Vander Borght TM, Albin RL, Gilman $\mathrm{S}$, et al. Presynaptic monoaminergic vesicles in Parkinson's disease and normal aging. Ann Neurol. 1996;40(6):873-84.

21. Beauchamp LC, Villemagne VL, Finkelstein DI, Doré V, Bush Al, Barnham $\mathrm{KJ}$, et al. Reduced striatal vesicular monoamine transporter 2 in REM sleep behavior disorder: imaging prodromal Parkinsonism. Sci Rep. 2020;10(1):1-7. https://doi.org/10.1038/s41598-020-74495-x.

22. Albin RL, Koeppe RA, Chervin RD, Consens FB, Wernette K, Frey KA, et al. Decreased striatal dopaminergic innervation in REM sleep behavior disorder. Neurology. 2000;55(9):1410-2.

23. Christopher L, Duff-Canning S, Koshimori Y, Segura B, Boileau I, Chen R, et al. Salience network and parahippocampal dopamine dysfunction in memory-impaired Parkinson disease. Ann Neurol. 2015;77(2):269-80.

24. Valli M, Cho SS, Masellis M, Chen R, Koshimori Y, Diez-Cirarda M, et al. Extra-striatal dopamine in Parkinson's disease with rapid eye movement sleep behavior disorder. J Neurosci Res. 2021;99(4):1177-87.

25. Evans AH, Katzenschlager R, Paviour D, O'Sullivan JD, Appel S, Lawrence $A D$, et al. Punding in Parkinson's disease: its relation to the dopamine dysregulation syndrome. Mov Disord. 2004;19(4):397-405.

26. Boeve BF, Molano JR, Ferman TJ, Smith GE, Lin S-C, Bieniek K, et al. Validation of the Mayo Sleep Questionnaire to screen for REM sleep behavior disorder in an aging and dementia cohort. Sleep Med. 2011;12(5):445-53.

27. Boeve BF, Molano JR, Ferman TJ, Lin S-C, Bieniek K, Tippmann-Peikert $M$, et al. Validation of the Mayo Sleep Questionnaire to screen for REM sleep behavior disorder in a community-based sample. J Clin Sleep Med. 2013;09(05):475-80.

28. Defer GL, Widner H, Marié RM, Rémy P, Levivier M. Core assessment program for surgical interventional therapies in Parkinson's disease (CAPSITPD). Mov Disord. 1999;14(4):572-84.

29. Nasreddine ZS, Phillips NA, Bedirian V, Charbonneau S, Whitehead V, Collin I, et al. The Montreal Cognitive Assessment, MoCA: a brief screening tool for mild cognitive impairment. J Am Geriatr Soc. 2005;53(4):695-9.

30. Beck A, Ward C, Mendelson M, Mock J, Erbaugh J. An inventory for measuring depression. Arch Gen Psychiatry. 1961;4:561-71.

31. Jewett DM, Kilbourn MR, Lee LC. A simple synthesis of [11C]dihydrotetrabenazine (DTBZ). Nucl Med Biol. 1997;24(2):197-9.

32. Rusjan P, Mamo D, Ginovart N, Hussey D, Vitcu I, Yasuno F, et al. An automated method for the extraction of regional data from PET images. Psychiatry Res Neuroimaging. 2006;147(1):79-89.

33. Studholme C, Hill DL, Hawkes DJ. Automated three-dimensional registration of magnetic resonance and positron emission tomography brain images by multiresolution optimization of voxel similarity measures. Med Phys. 1997;24(1):25-35.

34. Lammertsma AA, Hume SP. Simplified reference tissue model for PET receptor studies. Neuroimage. 1996:4(3):153-8.
35. Gunn RN, Lammertsma AA, Hume SP, Cunningham VJ. Parametric imaging of ligand-receptor binding in PET using a simplified reference region model. Neuroimage. 1997;6(4):279-87.

36. Mawlawi O, Martinez D, Slifstein M, Broft A, Chatterjee R, Hwang D-R, et al. Imaging human mesolimbic dopamine transmission with positron emission tomography: I. Accuracy and precision of $\mathrm{D}(2)$ receptor parameter measurements in ventral striatum. J Cereb Blood Flow Metab. 2001;21(9):1034-57.

37. Barnett V, Lewis T. Outliers in statistical data. 2nd ed. New York: Wiley; 1985. p. 1-608.

38. Cho SS, Christopher L, Koshimori Y, Li C, Lang AE, Houle S, et al. Decreased pallidal vesicular monoamine transporter type 2 availability in Parkinson's disease: the contribution of the nigropallidal pathway. Neurobiol Dis. 2019;1(124):176-82.

39. Martin W, Wieler M, Stoessl A, Schulzer M. Dihydrotetrabenazine positron emission tomography imaging in early, untreated Parkinson's disease. Ann Neurol. 2008;63(3):388-94.

40. Gilman S, Koeppe RA, Little R, An H, Junck L, Giordani B, et al. Striatal monoamine terminals in Lewy body dementia and Alzheimer's disease. Ann Neurol. 2004:55(6):774-80.

41. Bohnen NI, Albm RL, Koeppe RA, Wernette KA, Kilbourn MR, Minoshima $\mathrm{S}$, et al. Positron emission tomography of monoaminergic vesicular binding in aging and Parkinson disease. J Cereb Blood Flow Metab. 2006;26(9):1198-212.

42. Lung HJ, Weng Y-H, Wen M-C, Hsiao I-T, Lin K-J. Quantitative study of 18 F-(+)DTBZ image: comparison of PET template-based and MRI based image analysis. Sci Rep. 2018;8(1):1-10.

43. Okamura N, Villemagne VL, Drago J, Pejoska S, Dhamija RK, Mulligan $\mathrm{RS}$, et al. In vivo measurement of vesicular monoamine transporter type 2 density in Parkinson disease with 18F-AV-133. J Nucl Med. 2010;51(2):223-8.

44. Zarei M, Ibarretxe-Bilbao N, Compta Y, Hough M, Junque C, Bargallo N, et al. Research paper: cortical thinning is associated with disease stages and dementia in Parkinson's disease. J Neurol Neurosurg Psychiatry. 2013;84(8):875.

45. Cao R, Chen X, Xie C, Hu P, Wang K. Serial dopamine transporter imaging of nigrostriatal function in Parkinson's disease with probable REM sleep behavior disorder. Front Neurosci. 2020;14:349.

46. Kotagal V, Albin RL, Müller MLTM, Koeppe RA, Chervin RD, Frey KA, et al. Symptoms of rapid eye movement sleep behavior disorder are associated with cholinergic denervation in Parkinson disease. Ann Neurol. 2012;71(4):560-8.

47. German CL, Baladi MG, McFadden LM, Hanson GR, Fleckenstein AE. Regulation of the dopamine and vesicular monoamine transporters: pharmacological targets and implications for disease. Pharmacol Rev. 2015;67:1005-24

48. Sossi V, De La Fuente-Fernández R, Schulzer M, Troiano AR, Ruth TJ, Stoessl AJ. Dopamine transporter relation to dopamine turnover in Parkinson's disease: a positron emission tomography study. Ann Neurol. 2007;62(5):468-74.

49. De La Fuente-Fernández R, Sossi V, McCormick S, Schulzer M, Ruth TJ, Stoessl AJ. Visualizing vesicular dopamine dynamics in Parkinson's disease. Synapse. 2009;63(8):713-6.

50. Sommerauer M, Fedorova TD, Hansen AK, Knudsen K, Otto M, Jeppesen J, et al. Evaluation of the noradrenergic system in Parkinson's disease: an 11C-MeNER PET and neuromelanin MRI study. Brain. 2018;141(2):496-504.

51. Iranzo A, Santamaria J, Tolosa E. Idiopathic rapid eye movement sleep behaviour disorder: diagnosis, management, and the need for neuroprotective interventions. Lancet Neurol. 2016:15(4):405-19.

\section{Publisher's Note}

Springer Nature remains neutral with regard to jurisdictional claims in published maps and institutional affiliations. 